\title{
GESTIÓN POLÍTICA Y ETNICIDAD EN EL AYUNTAMIENTO DE SAN CRISTÓBAL DE LAS CASAS (1994-2008)
}

\author{
Emmanuel Nájera de León \\ emmanuelnaj@hotmail.com \\ Facultad de Ciencias Sociales \\ Universidad Autónoma de Chiapas
}

\section{RESUMEN}

Este trabajo tiene por objetivo analizar la participación de los funcionarios públicos indígenas — regidores - en la administración municipal de San Cristóbal de Las Casas.

Se pretende analizar el rol efectivo de dichos funcionarios en el ejercicio de las políticas locales, su influencia en procesos urbanos, y finalmente su papel y función dentro del cabildo municipal de San Cristóbal de Las Casas como parte del sistema de participación en la toma de decisiones.

El estudio se circunscribe al período 1994-2008. En un primer momento, se presenta parte de la reflexión teórica sobre el concepto «etnicidad», posteriormente se ofrece un marco sociohistórico de la trayectoria reivindicativa étnico-indígena en el contexto urbano sancristobalense —transición en las demandas, las estrategias y las formas de negociación-, y en la última parte se da cuenta de la construcción sociohistórica de la participación política indígena dentro de la lógica administrativa municipal. Finalmente se ofrecen algunas reflexiones.

Palabras clave: etnicidad, luchas reivindicativas, funcionarios indígenas, gobiernos municipales, gestión política. 


\section{ABSTRACT}

This work aims to analyze the actual participation of indigenous public officials councilors- within the municipal administration of San Cristóbal de Las Casas.

It attempts to analyze the effective role of these public officials in the implementation of local policies, their influence on urban processes and their role and function within the municipal council of San Cristóbal de Las Casas as part of the effective system of participation in the decision-making process.

The study is circumscribed to the period from 1994 to 2008. In a first moment it presents part of the theoretical reflection around the notion of «ethnicity». It later offers a sociohistorical framework of the trajectory of ethnic-indigenous demands in the urban context of San Cristóbal de Las Casas - transition in the demands, strategies and forms of negotiation. The last part describes the socio-historical construction of indigenous political participation within the logic of municipal administration. Finally, some reflections are offered.

Key words: ethnicity, struggles for indigenous demands, indigenous state officials, municipal governments, policy management. 


\section{INTRODUCCIÓN}

En los últimos años, el estudio de la participación de líderes indígenas en los gobiernos locales ha cobrado importancia. Dicho interés, propio de los años noventa (Assies y Gundermann 2007: 11), va a la par con el florecimiento a nivel global de los debates sobre la ciudadanía, la diversidad cultural, la exclusión y los derechos, desde los más universales, los derechos humanos, hasta los más específicos de carácter nacional, pasando por los sociales y políticos.

A decir de Bengoa, esto se relaciona con el fin del primer ciclo, que llamó de emergencia indígena —es decir, la presencia de nuevas identidades y expresiones étnicas que se han acompañado de acciones, reivindicaciones y reclamos de las poblaciones indígenas - y la apertura de un segundo ciclo, iniciado con la experiencia de gobierno de Evo Morales en Bolivia a partir de 2006 y la llegada de líderes de una variedad de organizaciones indígenas a las instituciones del Estado o a los órdenes de gobierno local (2009: 7)

En el terreno particular de los movimientos étnico-indígenas, hay que señalar que la trayectoria de las «luchas», atestiguada en los últimos años en varias regiones, se ha caracterizado por una reorientación que, a decir de Clair, demuestra una tendencia hacia la reivindicación del «ejercicio de la ciudadanía» (1995: 155), caracterizada ésta por el énfasis en un conjunto de demandas y prácticas políticas que apelan a la profundización de la democracia, a la participación política efectiva (Stavenhagen 2003), a la posibilidad de acceder al espacio público (Martínez 2008: 81) y, sobre todo, a la búsqueda del reconocimiento de derechos económicos, sociales, culturales y políticos por parte de los Estados nacionales.

Recientemente, Assies y Gundermann (2007) y Obregón (2003) han observado que la población indígena ha ido ganando experiencia en su relación con los partidos políticos e incluso ha participado más en las propias instituciones políticas (Freidenberg 2004: 102). Sin embargo, la documentación de estas «nuevas prácticas» en la mayoría de los casos ha 
sido de orden cuantitativo, sin reflexión cualitativa. Se ha dejado de lado el análisis de las múltiples estrategias construidas por la población indígena para incorporarse a los gobiernos locales y, lo más importante, su rol efectivo para gestionar la agenda política una vez situados en los ayuntamientos.

Considerando lo anterior, resulta importante comprender las experiencias de participación de líderes, organizaciones y del mismo movimiento indígena en gobiernos locales urbanos con el fin de explorar los alcances y limitaciones de esta presencia, así como los desafíos que plantean tanto para la configuración y la institucionalidad política local como para los propios líderes una vez situados en los gobiernos municipales.

\section{LA ETNICIDAD COMO ESTRATEGIA: CLAVE PARA ENTENDER LA CUESTIÓN INDÍGENA}

El término «etnicidad», frecuentemente usado en las ciencias sociales, según Wade se ha tratado en muchas ocasiones como sinónimo de la noción «raza», palabra que ha sido considerada per se como un propagador del racismo (Wade 2000: 16). Por otra parte, Gall (2003) comenta que, a pesar de que el término «etnicidad» es relativamente nuevo, en muchas ocasiones se ha empleado vagamente, a veces para designar lo mismo que la noción «raza» pero con connotaciones menos emotivas.

No obstante sus posibles aplicaciones teóricas, el concepto sigue «encuadrando» a los estudiosos hacia dos perspectivas. En primer lugar hacia la visión primordialista, sentada en las bases de los enfoques culturales ortodoxos, la cual sostiene que la etnicidad «opera» como una cualidad primordial, esto es que existe de forma natural, desde siempre, y por lo tanto es propia de la existencia humana (Smith 1997: 18). De esta manera, la etnicidad operaría como un atributo fijo dentro de las colectividades sociales fundamentándose en los aspectos culturales previamente establecidos —el parentesco, la lengua, la religión y las costumbres de los pueblos - que determinarían la identidad cultural de cada uno de los miembros. 
Desde esta visión, los grupos étnicos se concebirían como unidades discretas y definibles taxonómicamente, en buena medida, mediante un listado de rasgos culturales observables que delimitarían dicha presencia. Sin embargo, esta idea muestra únicamente, como bien alude Río, «una forma de identificación primaria inflexible y todopoderosa que da lugar a comunidades naturales antes que a comunidades de intereses» (2002: 86), por lo que se estaría obviando el carácter dinámico, si cabe el término, de los escenarios construidos en los últimos años tanto por los movimientos étnico-indígenas como por las propias experiencias de organización india en la escena rural y en la urbana.

Efectivamente, tanto la propia adaptación que surge de las negociaciones de los individuos con las condiciones simbólicas y materiales de su entorno (Terrén 2002: 46), como las transformaciones selectivas construidas en función de su eficacia estratégica, quedarían ensombrecidas bajo un modelo que delimita de forma rígida y esencialista las relaciones intragrupales e intergrupales, oda vez que, las etnias pasarían, como bien indica Giménez, a considerarse como entidades culturales discretas y preconstruidas, definibles en sí mismas a partir de las características intrínsecas de los grupos y las personas pertenecientes a esos grupos (2006: 131).

De manera opuesta, la visión constructivista o instrumentalista, sentada en la propuesta de Barth (1976) y en la sociología de las relaciones étnicas (Terrén 2002), sostiene que la etnicidad, lejos de reducirse a un hecho dado de la existencia social, aparece dentro de un escenario abierto, diverso y complejo de identidad y sentimiento de pertenencia, lo que exige una concepción más compleja y multidimensional del funcionamiento de la cuestión étnica «en el marco de flexibles procesos de organización y definición política de las diferencias culturales» (Río 2002: 88). En ese sentido, la etnicidad asumiría un carácter situacional toda vez que, como indica Smith:

La pertenencia a un grupo étnico es una cuestión de actitudes, percepciones y sentimientos en que se encuentra el sujeto: a medida que va cambiando la situación del individuo, 
también cambia la identificación del grupo, o por lo menos, la importancia de las identidades y discursos a las que se adhiere el individuo irá variando conforme pase el tiempo y las situaciones cambien (1997: 18).

Desde esta perspectiva, la noción de etnicidad permitiría comprender el carácter dinámico y situacional de los grupos étnicos y sus diferencias culturales. Dicho sea de paso, estas diferencias, lejos de manifestarse de manera aislada, se mostrarían en la medida que se construyeran escenarios de contacto e interacción social. De esta manera, se asociaría la etnicidad a un ejercicio de definición de categorías étnicas desde una posición relacional, «lo que implicaría un encuadre situacional-relacional de identidad y diferencia más que la manifestación de un inmanente ser biológico o cultural» (Restrepo 2004: 17).

Ciertamente, como bien observó Río, los grupos étnicos se considerarían, antes que comunidades naturales — guiadas por rígidos principios de identificación e interacción—, actores sociales que perciben y hasta producen sus igualdades y diferencias étnicas mediante el contacto con otros grupos dentro de un orden de interacción intergrupal en el que se desarrolla un repertorio flexible de estrategias identitarias para maximizar el valor político de ciertos indicadores culturales (2002: 88).

No obstante dicha trayectoria epistemológica, las preguntas serían, ¿qué quiere decir concretamente el término «etnicidad»? y ¿qué implica hablar de etnicidad hoy? Se puede decir, en un primer momento, que la noción de etnicidad es una construcción social que se refiere fundamentalmente a las identificaciones de diferencia y similitud (Gall 2003: 76) y, por lo tanto, no a una realidad dada natural, inevitable e inexplicablemente sino producto de un proceso dinámico de construcción social, histórica y política (Gutiérrez 2008: 22). Es decir, a final de cuentas la etnicidad es el resultado de esfuerzos conscientes por parte de los individuos y grupos para movilizar símbolos étnicos y acceder, mediante ese esfuerzo de politización de las diferencias culturales, a recursos sociales, políticos y materiales (McKay 1982: 399, citado en Río 2002: 90), por lo que lejos de ser algo fijo o una esencia, 
ésta se muestra como un proceso en perpetua construcción (Assies y Gundermann 2007: 20).

De estas ideas se puede desprender que la pertenencia étnica cambia situacionalmente, se recrea y se reinventa según las necesidades del grupo. Por lo tanto, es posible pensar que la definición del «nosotros», —la auto-percepción- implica una definición del grupo que cambia en el contacto con la diferencia y se define situacionalmente dependiendo de los distintos «otros»/«ellos». De esta manera se puede decir que la etnicidad y las categorizaciones étnicas tendrían que reflexionarse a partir de escenarios particulares y tiempos y contextos específicos, es decir, como parte de una historia específica (Wade 2000: 17-19).

Asimismo, tal como afirma Gutiérrez, la constitución de la etnicidad como un aspecto de las relaciones sociales que surge entre actores sociales que se consideran y son considerados por los demás como culturalmente distintos de otros grupos, implica abrir el camino hacia los movimientos políticos, sociales y culturales que revalorizan en el espacio público todo aquello que concierne a la diversidad de grupos de pertenencia (2008: 19).

Por lo tanto, la pregunta a contestar después de este recorrido teórico sería, ¿qué implica hablar de etnicidad en el mundo actual? Sin el afán de ofrecer una respuesta acabada, se puede afirmar inicialmente que la etnicidad forma parte de la construcción de una identidad genérica y, como alude Gros, lejos de reflejar un rechazo de la modernidad y un repliegue comunitario, se representaría como un «recurso» accesible y fuertemente instrumentalizado en pro de una integración (1998: 181) o reivindicación en el espacio público. De ahí que sea necesario situar esta noción en los nuevos escenarios que la realidad nos ofrece. 


\section{EL PRIMER CICLO DE LA «EMERGENCIA ÍNDIGENA»: ENTRE LA REPRESENTACIÓN NEGADA Y LA LUCHA POR EL DERECHO A LA CIUDAD}

Al inicio de los años noventa, la lucha por el derecho a la ciudad de la población indígena en San Cristóbal de Las Casas se hizo más intensa, a pesar de que ya existían organizaciones como el Consejo de Representantes Indígenas de los Altos de Chiapas (CRIACH) y la Organización Regional de Indígenas de los Altos de Chiapas (ORIACH). Esta última fungió a mediados de los ochenta como principal interlocutora entre los indígenas expulsados y el gobierno del estado para tratar de poner fin al escenario conflictivo alteño. Asimismo, ya para entonces la ORIACH había integrado entre sus reivindicaciones la situación por la que atravesaban las «nuevas» colonias indígenas.

En la praxis política, la ampliación de la agenda reivindicativa indígena incluyó la construcción de un presupuesto «bajo el signo de cuestiones culturales y étnicas» (Le Bot 2008: 83) que actuó como motor de acción en el proceso de gestión En algunos casos, la estrategia incluyó la reivindicación de la ciudadanía como sentido de la pertenencia de los indígenas a la ciudad coleta $^{1}$, por lo que en varias ocasiones el discurso se orientó hacia la reivindicación de su residencia en la ciudad dado que llevaban viviendo en ella más de veinte años, lo que les otorgaba el derecho, según estos dirigentes, a mejores condiciones en el municipio. En otros casos, la lógica organizativa enfocó su estrategia en el cabildeo con las autoridades municipales buscando la provisión de servicios públicos.

A mediados de 1990, la problemática urbana se acrecentó. Las demandas emanadas de la población indígena continuaron, si bien la estrategia siguió enfocada en el diálogo con el gobierno local por medio de las organizaciones históricas CRIACH y ORIACH. A esta última se integraron representantes de las colonias indígenas — La Hormiga, Tlaxcala, La Quinta, Diego de Mazariegos, Morelos, Getsemaní, Jerusalén- cuyo discurso se dirigió a rescatar la cualidad ciudadana y el derecho a mejores condiciones en estas nuevas «áreas» de la ciudad a pesar de ser considerados asentamientos «irregulares». En una carta dirigida 
al gobierno municipal del Ing. Rodríguez (1989-1991), la ORIACH y algunos colonos indígenas señalaron lo siguiente:

los que suscribimos como representantes de las diversas colonias del mismo municipio, nos permitimos dirigirnos a usted para solicitarle una reunión urgente en la Col. Getzemaní... los habitantes de estas colonias tenemos un sin número de necesidades de primer orden para la vida de cualquier ser humano: i) la introducción del agua potable ii) construcción de un aula escolar iii) construcción de una cancha deportiva iv) respeto a la propiedad privada v) posibilidad de proyectos productivos y ocupación de mano de obra.

\section{Y agregan:}

Sabemos que somos nosotros los principales protagonistas del cambio, pero entendemos que con la ayuda de nuestras autoridades lo lograremos más pronto, por bien propio de nuestras familias y de nuestra querida patria...sabemos que usted es una persona que se interesa por los problemas de su pueblo y busca solventarlos a la medida de sus posibilidades, rogámosle para que los nuestros tengan una solución.

Aunque el escenario organizativo étnico-indígena supuso la construcción de estrategias que fungieron como un recurso movilizador en pro del derecho a la ciudad, hay que enfatizar que esta lógica no fue lineal ni obtuvo resultados inmediatos. A pesar de utilizar el cabildeo como estrategia para la integración a la dinámica de la ciudad, hay que señalar que durante los años noventa la cuestión de la regulación y la implementación de servicios públicos continuó en la misma ruta: con una intervención de la administración local tardía y a cuenta gotas en la decena de colonias indígenas.

No obstante dicho escenario, en agosto de 1990 la administración del Ing. Rodríguez, junto con el gobierno estatal de González Garrido (1988-1993), implementó el «Programa de regularización de colonias populares». Aunque este programa fue reconocido por sus bondades y por representar un hecho histórico para el municipio coleto, debe señalarse que 
en más de un año de implementación sólo logró cubrir cuatro colonias de los más de 45 asentamientos irregulares existentes en la cabecera de San Cristóbal de Las Casas. A ellas se integraron diez colonias más que tenían la intención de regularizarse, pero al no cumplir algunos requisitos, como el de ubicarse en zonas aptas para vivienda, e incluso por el mismo proceso burocrático, no entraron al programa de tenencia de la tierra urbana.

A pesar del proceso de incorporación de las colonias a la otrora ciudad mestiza, la base organizativa indígena continuó con su demanda de dotación de servicios públicos para los demás asentamientos. Si bien con una base organizativa incipiente, en la mayoría de los casos bajo los lineamientos de las organizaciones históricas CRIACH y ORIACH, el discurso indígena supuso «una apropiación y reapropiación de los discursos y símbolos desplegados» (Bello 2004: 17), además de la incorporación de una variedad de elementos -la representación de la pobreza, la marginalidad y la poca atención hacia las colonias indígenas del centro urbano, además del discurso ciudadano indígena - que se convirtieron en el motor de actuación de la base organizativa indígena en el centro urbano.

$\mathrm{Al}$ inicio de los años noventa, la representación indígena en el ayuntamiento era un tema impensable. A pesar de que las administraciones ya habían abordado el «reconocimiento» de la población india como ciudadanos sancristobalenses, no había presencia indígena en los espacios de decisión local, ni en los partidos políticos ni en la configuración de planillas municipales.

Pese a su «ausencia» en los espacios de representación, en la práctica la población indígena construyó estrategias que le permitieron mantenerse dentro de la dinámica urbana e involucrarse poco a poco en la arena político-electoral local. Aunque este proceso de incorporación no se dio de la noche a la mañana, al inicio de los años noventa surgieron múltiples escenarios que atestiguaron la presencia indígena en la escena política local.

En el ámbito de las colonias indígenas, fuesen regulares o irregulares, los representantes de 
los concejos vecinales —órganos de intermediación entre el ayuntamiento y la ciudadanía en los procesos de gestión - y los propios procesos organizativos de gestión indígena se convirtieron en los principales «promotores» del voto priista. Esta mecánica organizativa permitió a los líderes indígenas «negociar» el voto indio a cambio de la regularización de predios, la gestión de obras de infraestructura, la introducción de servicios públicos —agua, drenaje, energía eléctrica — y, tiempo después, espacios dentro del ayuntamiento coleto.

Esta lógica de organización se complementó con la conformación de los llamados comités seccionales impulsados por el PRI. Dichos comités, que pretendieron ser espacios de representación y gestión de las necesidades vecinales -barrios, colonias y fraccionamientos—, terminaron por situarse como espacios de representación y «gestión» del voto en cada uno de los barrios y colonias de San Cristóbal durante los procesos electorales. Como señala un ex regidor:

...bueno, pues fue por invitación de otros militantes. Empezamos en la misma colonia, de que sí queríamos apoyar formando parte de algún comité seccional o promotores. De esa manera iniciamos las comisiones, llegaron las personas, los líderes y representantes y también ahí como promotores o como presidentes seccionales, existe la oportunidad de que trabajes para hacer gestión a favor de los militantes y de tus vecinos, entonces de esa manera pues vas conociendo y metiéndote dentro del partido para que de alguna manera vayas conociendo cuál es la estructura para trabajar para tu comunidad ${ }^{2}$.

Aunque, a decir de los representantes indígenas, esta dinámica organizativa les permitió ser reconocidos como ciudadanos sancristobalenses y trabajar por las necesidades vecinales, la incorporación de representantes indios a la iniciativa priísta y posteriormente a otros partidos políticos terminó por reducirse a una mera transacción del voto, muchas veces a costa del discurso de la regularización de predios o la gestión de servicios públicos, cartas importantes para las instituciones políticas durante los procesos electorales locales.

Otro escenario que poco a poco permitió una redefinición de la presencia indígena en la 
escena política local fue la disputa y el control étnicamente diferenciado de los mercados y plazas públicas. A partir de los años ochenta, a decir de Hvostoff (2004), el comercio se indianizó. A los monopolios que ya habían adquirido en la ciudad — venta y trabajo de la madera, venta de la juncia, legumbres, flores, helados, voceadores, boleadores-, la población india agregó una competencia fuerte en otro tipo de negocios tales como las artesanías o el transporte público.

Esta presencia indígena, respaldada por una estructura corporativa dividida pero muy eficaz, ofreció resultados inmediatos, uno de ellos, la conquista de nuevos espacios dentro del mercado municipal Castillo Tielmans y el mercadito II. A mediados de los años noventa, esta reorganización sistemática, como parte de nuevos procesos de diferenciación y de las reivindicaciones históricas indias, logró penetrar el tozudo control mestizo de los sindicatos de comerciantes. Por lo tanto, a la dirección de los sindicatos casi exclusivamente controlados por ladinos —la Confederación Nacional Campesina (CNC), la Confederación Revolucionaria de Obreros de México (CROM), la Confederación de Trabajadores de México (CTM) o la Unión Sindical de Locatarios (USLO)— poco a poco se presentaron aspiraciones indias.

En todo este proceso se observó un esfuerzo de la población indígena por tener presencia en espacios económicos locales que tradicionalmente habían sido ocupados por los gremios ladinos. Esta dinámica, que ganó más adeptos después de la irrupción armada de 1994, modificó los patrones de mediación y las redes tradicionales de poder. La población indígena pasó de una interlocución pasiva dirigida por líderes gremiales mestizos a convocar procesos organizativos precedidos por mediaciones directas con el Estado.

A pesar de esta dinámica organizativa y del discurso de los gobiernos municipales coletos, que ofrecían la integración de la población indígena y simultáneamente no consideraban sus reivindicaciones en las agendas de gobierno, la presencia india en la escena política fue poco a poco ganando terreno a medida que los «representantes» indios se posicionaron 
como interlocutores legítimos de las gestiones vecinales, especialmente después de la irrupción armada zapatista. Sin embargo, la clase política mestiza se resistía a incluirlos en las planillas municipales so pretexto de la no vecindad de muchos indígenas en San Cristóbal.

Con todo ello, después de la irrupción armada de 1994, la presencia indígena en la arena política tuvo diversos matices. Aunque los discursos políticos tanto de las administraciones municipales como de los partidos políticos enfatizaban la cualidad multicultural del centro urbano de San Cristóbal, la representación indígena en la lógica política continuó reducida a la mera «gestión» del voto indio muchas veces a cambio de mejoras a cuentagotas en las colonias indias. Sin embargo, esta dinámica también fortaleció la presencia de los representantes indígenas — presidentes de colonias— en la arena político-electoral.

Durante la gestión del Lic. Rolando Villafuerte Aguilar (1996-1998), la administración local continuó conformada por una plana mestiza prísta, muchos procedentes de los sindicatos locales que se habían convertido en el granero de votos del partido oficial. También, por primera vez se integraron las representaciones del PAN y del PRD. Este último partido incorporó al indígena Mariano de la Cruz Ruiz, conocido comerciante migrante con presencia en el mercado de las artesanías y que «representaba» a una parte de la población india asentada en el centro urbano.

La administración de Villafuerte Aguilar sostuvo un discurso político de reconocimiento hacia la población indígena asentada en el periférico norte. Durante la etapa de campaña, y después como representante del municipio coleto, fue enfático en la complejidad que ofrecía la escena sancristobalense y en la urgente necesidad de tomar en cuenta las necesidades de los ciudadanos de la zona norte. Este reconocimiento en gran medida se había concretado por la escena convulsa de 1994 y por la presencia más visible de instancias de los gobiernos federal y estatal, que obligaron a las administraciones priístas a iniciar fuertes procesos de incorporación de los habitantes indígenas a la dinámica urbana, 
lo cual se concretó en la regularización de predios y en la provisión de servicios públicos. Sin embargo, en el ambiente político-local, la incorporación del indígena Mariano al ayuntamiento de Villafuerte Aguilar contribuyó más al posicionamiento del PRD como una opción política dentro de la población indígena, que a un proceso de visibilización de las reivindicaciones indias en San Cristóbal.

Ciertamente, pese a que el PRD había incorporado al líder indígena artesano a su facción, y pese a haberse posicionado durante la campaña como la fuerza política que contemplaba las reivindicaciones indias de ciudadanía en el centro urbano no las consideró posteriormente en la agenda gubernamental. Más bien al contrario, muchas veces la presencia indígena en el PRD se redujo a la reproducción de la vieja estructura del priísmo local, que veía en la población indígena una «cartera» importante de votos.

Asimismo, la presencia de Mariano tampoco implicó un posicionamiento de la población indígena en los procesos decisorios del ayuntamiento, toda vez que su participación se redujo a la integración de comisiones municipales — mercados y centros de abasto, comercio, turismo y artesanías, y la comisión de adquisiciones, arrendamientos y servicios-, comisiones que normalmente estaban precedidas por la plana mestiza. A pesar de lo anterior, aunque la presencia indígena no se materializó en la toma de decisiones municipales, sí contribuyó a que se construyeran puentes para mantener la mediación entra la población india y el municipio.

\section{LA INCLUSIÓN INDÍGENA EN EL AYUNTAMIENTO COLETO}

A finales de los años noventa, la inclusión de líderes indígenas en las planillas locales fue una realidad. Si bien dicho ejercicio se consolidó en la medida en que el tema indio se posicionó en Chiapas —especialmente por la irrupción armada de 1994 y los diálogos de 1996 en San Andrés Larráinzar-, fue en 1998 cuando la demanda por la incorporación indígena a la arena político-electoral y la participación en el proceso de toma de decisiones 
se incluirían en la reforma electoral chiapaneca.

Ciertamente, la reforma electoral de abril de ese año —que dicho sea de paso retomó una de las reivindicaciones de los acuerdos de San Andrés- invitó a los partidos políticos a que incluyeran preferentemente candidatos «ciudadanos» indígenas en puestos de representación — congreso local y planillas municipales - para los municipios donde la población fuese mayoritariamente india, augurando, por lo menos en el discurso, una mayor presencia india en la dinámica electoral chiapaneca.

En el caso sancristobalense, las primeras medidas impulsadas por los partidos políticos se orientaron hacia el reconocimiento de la ciudadanía de la población india, aunque este discurso ya había sido empleado por la administración de Villafuerte Aguilar (1996-1998). En los procesos electorales, dicha bandera fue compartida por las diversas fórmulas políticas a pesar de la continuidad — en la lógica política mestiza - de un discurso que caminó por una frontera muy delgada entre el reconocimiento y la omisión de las reivindicaciones indias con el pretexto de la no vecindad de esta población.

No obstante dicho escenario, en la praxis política y en el propio ejercicio de toma de decisiones los funcionarios indígenas continuaron con un espacio muy reducido. A pesar de que varios partidos políticos —PRI, PRD, el Partido del Trabajo (PT), PVEMenfatizaron la urgente necesidad de integrar a los diversos «sectores» de la población sancristobalense en la toma de decisiones, fue común que los espacios «ganados» en las elecciones locales continuaran siendo ocupados por la plana mestiza; o en su caso se incluyera a líderes indígenas, fundamentalmente del área rural, con la finalidad de reproducir prácticas poco ciudadanas como la cooptación y el clientelismo político al viejo estilo priísta.

Por ejemplo, durante la administración del Ing. Mariano Díaz Ochoa (1999-2001) se incorporó a la plana municipal el regidor Manuel Pérez López, representante de la zona 
rural. Si bien dicha designación constituyó un nuevo escenario dentro de la administración municipal, igualmente no significó una mayor presencia del tema indígena al interior de la administración sancristobalense, sino que ésta se redujo a la diversificación de la mecánica política priísta y a una mayor presencia del prí́smo coleto en el área rural del municipio y entre la población indígena del área urbana.

A ello hay que añadir que el regidor Manuel Pérez López se incorporó a un par de comisiones municipales, la de salubridad y asistencia social, y la de industria, comercio, turismo y artesanías. Sin embargo, las sesiones de cabildo consultadas dan cuenta de una invisibilidad del regidor Manuel en los debates de tales comisiones, estando las resoluciones finales a cargo de la plana mestiza.

Por ese mismo camino transitó la inclusión de funcionarios indígenas en la gestión del primer gobierno municipal surgido de una fuerza política distinta al PRI. Si bien la administración del Lic. Hernández Cruz (2002-2004) hizo énfasis en la necesidad de construir una «nueva» relación con la población indígena basada en el respeto hacia la diversidad cultural, el reconocimiento de la vecindad indígena en el centro urbano coleto y la integración de población indígena en espacios de representación y toma de decisiones, la incorporación indígena continuó siendo poco significativa.

La estrategia abanderada por la administración de Hernández Cruz en el tema de representación indígena consistió en incorporar a indígenas de diversas áreas del municipio de San Cristóbal. Tras Catarino Ruíz Jiménez, representante del ejido La Albarrada, se incorporó el indígena Sebastián López López, reconocido abarrotero que hacía tiempo radicaba en la ciudad como empresario. Además, a la misma representación política PAS - se integró el indígena Mateo Hernández López, reconocido comunicador de la otrora radio comunidad indígena que se distinguió por su capacidad de gestión y acompañamiento a las demandas indígenas a nivel regional y local. 
Con presencia indígena tanto en la plana municipal como en la arena político-local, además de los discursos nacional y estatal que ponderaban la nueva escena pluricultural chiapaneca, la lógica de la incorporación y representación política de la población india pareció situarse, por lo menos en el escenario sancristobalense, en la misma dinámica de las administraciones priístas aunque con un tono que pretendió ser diferente, reconociéndose ahora la cualidad ciudadana y el derecho de representación indígena en la toma de decisiones.

Durante la administración de Hernández Cruz, la representación indígena ocupó la presidencia de un par de comisiones municipales. Catarino Ruiz, representante del ejido La Albarrada y principal interlocutor de la zona rural coleta, presidió la comisión de participación comunitaria, organismo que se erigió como el principal puente de comunicación entre el ayuntamiento y el área rural e indígena del municipio. Por su parte, el indígena Sebastián López López presidió la comisión de mercados y centros de abasto. Dicho cargo se había convertido durante las administraciones priístas en uno de los pilares del voto corporativo de los sindicatos locales y permitía la legitimidad de los dirigentes frente a sus agremiados siempre que les permitieran ubicarse para la venta en los mercados municipales, las plazas públicas e incluso en las aceras cercanas a los espacios comerciales a pesar de la «ilegalidad», los conflictos entre locatarios y los costos que muchas veces eso significó para las administraciones coletas.

Además de ocupar las presidencias de comisiones indicadas, durante la gestión de Hernández la representación indígena también formó parte de otras comisiones municipales al igual que en las administraciones priístas. Catarino Ruíz formó parte de la comisión de industria, comercio, turismo y artesanías, la comisión de seguridad pública y la de ambulantaje. Por su parte, el regidor Sebastián López participó en la comisión de limpia, la de salubridad y asistencia social, y la comisión de obras, adquisiciones, arrendamientos y servicios. 
Si bien la presencia indígena en el PAS implicó una mayor participación numérica en la administración local, esto no significó que los regidores indígenas realmente participaran en la toma de decisiones. Por el contrario, al igual que durante las gestiones priístas, la administración de Hernández Cruz les ofreció pocos espacios en los procesos de debate y en la gestión de la agenda gubernamental.

Conforme la gestión de Hernández Cruz avanzó, los regidores indígenas pocas veces presentaron iniciativas en las diversas comisiones y su participación en los debates, según dan cuenta las sesiones de cabildo, fue prácticamente nula.

\section{ENTRE RESISTENCIAS Y REALIDADES: FUNCIONARIOS INDIOS Y GESTIÓN POLÍTICA EN SAN CRISTÓBAL (2005-2008)}

Durante la gestión tricolor del Lic. Sergio Lobato García (2005-2007), los dirigentes indios volvieron a situarse en la administración local. Aunque al principio esto no resultó novedoso dado que en las planillas locales anteriores había sido común la inclusión de la representación rural, lo interesante fue que dicha gestión se convirtió en la primera administración municipal integrada por cinco regidores indígenas, entre propietarios, suplentes y plurinominales.

Los tres espacios del PRI fueron ocupados por representantes indios pertenecientes a la llamada Coordinación de Colonias de la Zona Norte (CCZN) —espacio de gestión que impulsaba las demandas vecinales frente a las administraciones municipales y que hasta mediados de los años noventa había obtenido algunos resultados- En ese trío figuraba como tercer regidor el Prof. Antonio García González, militante priísta originario del municipio de Huixtán pero avecindado en el valle coleto desde hacía más de cuatro décadas. Había hecho carrera en el magisterio rural y, tras instalarse en la llamada zona norte en 1979, se convirtió en uno de los fundadores de la iniciativa gestora de la CCZN. 
Otro de los representantes indígenas de la plana tricolor fue el Ing. Filiberto Fermín Gómez Rodríguez, octavo regidor, reconocido por la población india debido a su labor a nivel local y regional en el Centro de Desarrollo Comunitario «La Albarrada» - hoy denominado Comunidades de Aprendizaje para el Desarrollo «Porfirio Encino Hernández, La Albarrada»—. En el partido, el Ing. Gómez Rodríguez era identificado por su labor proselitista en los comités seccionales — gestión del voto— de las colonias del norte de la ciudad, además de por encabezar la CCZN al momento de su designación. Con ellos se integró Andrés Gómez Hernández como cuarto regidor suplente.

Estas incorporaciones reconfiguraron la trayectoria india en la dinámica política coleta. Como punto fundamental, se reconoció la presencia vecinal del norte de la ciudad, vía representantes de colonias, quienes después de 1994 fungieron como los principales intermediarios de las demandas vecinales frente al ayuntamiento. Aunque esta dinámica política muchas veces otorgó visibilidad a los representantes indios en el entramado político-local, no implicó necesariamente mayores espacios dentro de los partidos políticos ni mucho menos protagonismo en el proceso de toma de decisiones del ayuntamiento.

Ciertamente hay que enfatizar que, tras la «presencia» india, se hallaban escenarios de negociación y transacción del voto, y además había divergencias entre los propios líderes indígenas que, al observar los «privilegios» conseguidos en la función pública, generaron encono y división al interior de las iniciativas de gestión indígena en la ciudad. A decir del propio Filiberto Fermín Gómez, esto era común al aproximarse los comicios municipales:

La conclusión a la que se llegó fue de que se juntara a toda la gente [refiriéndose a los militantes indios del PRD, PRI, PAN] y que no nos fijáramos en el partido; así se eligieron a dos o tres candidatos que pudieran ocupar la regiduría; nuestra propuesta se presentó a todos los candidatos municipales tanto al Licenciado Sergio, a Salvador Camacho, estaba también Rubisel Guillén y a otro partido. Se formó una comisión negociadora que entraron en pláticas con los candidatos para incluir a los candidatos de la zona norte. Digamos que el que tuvo mayor visión fue el licenciado Sergio, aceptando las tres personas que propuso la 
zona norte. Entonces creo que vamos cambiando la forma de pensar, de pedir nuestras cosas y de participar en la política por lo menos de otra manera, ya no de golpe, sino a través del diálogo y de mayor presencia en la presidencia municipal. ${ }^{3}$

En palabras del Ing. Gómez, este «logro» político se relaciona con los caminos construidos por la población india en la ciudad. Aunque no lo señalé explícitamente, todo el proceso mencionado ha implicado la utilización de la identidad como moneda de cambio, lo que en última instancia ha producido buenos dividendos a las dirigencias indias y en menor medida a la población que representan. A ello habrá que agregar que el aumento de la visibilidad de las dirigencias indias y de su capacidad para aglutinar a sus «representados» implicó una mayor presencia de los líderes vecinales en el ambiente político-local.

También el discurso de Filiberto Fermín Gómez da cuenta de cómo los procesos de construcción de ciudadanía indígena se han reducido a una mera transacción del voto, muchas veces a cuenta de mejoras vecinales. Esto no pudo comprenderse enteramente hasta 1995, dado que la relación entre las administraciones municipales y la población indígena estuvo atravesada por la invisibilidad de esta última e incluso por la «resistencia» de las gestiones coletas a permitirles una participación real.

A la facción del PVEM, que había obtenido cuatro espacios en la administración coleta, se integró el comunicador indígena Mateo Hernández López, antiguo militante del PAS y que, debido a la pérdida de registro de dicho partido, decidió vincularse a las filas del partido verde apoyando la candidatura del locutor mestizo Salvador Camacho Corzo. Dada su historia de acompañamiento a los procesos de gestión de varias iniciativas indígenas en la región, la militancia de Mateo Hernández en el partido ecologista tenía como principal objetivo la continuidad en las tareas de gestión a favor de la población india, además del apoyo al discurso en torno al cuidado del ambiente impulsado por dicha opción política.

Sí vi que muchos partidos empezaron a buscarme, tal vez porque sí me conoce mucha gente. Ya después me buscó el PT, el PRD, el PAN incluso. El único que no me buscó 
sinceramente fue el PRI, pero al analizar todas estas invitaciones no me convencían por sus estatutos, pero al ver al Partido Verde vi que era para proteger el medio ambiente y analizándolo bien me gustó ese partido. Ya fue que ahora si yo fui a hablar, fuimos con Salvador y hablamos, que la estructura del partido pues como que era conveniente y ellos también sabían más o menos quiénes éramos. Ya fue que se nos aceptaron con buena voluntad y con buena intención y hasta ahorita seguimos participando en este partido y creo que de ahí pues no nos cambiaremos mientras no se pierda el registro como pasó en el PAS.

Si bien el planteamiento de Mateo Hernández parece referirse a un verdadero proceso de integración indígena en la escena político-electoral y en los espacios de representación política, hay que subrayar que, hasta finales de los años noventa, y pese a las reformas constitucionales y electorales en materia de representación indígena, los avances se redujeron al aparente cumplimiento de dichas reformas jurídicas y no a la verdadera construcción de una ciudadanía indígena visibilizada tanto en la «competencia» dentro de los partidos políticos como en el proceso de toma decisiones de las administraciones municipales.

Finalmente, el último espacio lo ocupó el indígena Agustín Gómez Patishtán, quien, tras haberse formado en el perredismo local, se convirtió durante el proceso electoral local de 2004 en el primer candidato indígena que contendió para la presidencia municipal bajo las siglas del Partido Convergencia, Sin embargo, diferencias «político-ideológicas» al interior del partido ocasionaron fuertes disputas entre los militantes mestizos y los dirigentes indios que echaron por tierra las demandas de líderes indígenas para incorporarse a espacios de representación.

Gómez Patishtán había participado en el ámbito de las organizaciones indígenas a nivel regional reivindicando límites territoriales y mejores condiciones productivas, al igual que mediando en problemas ejidales. Además, había trabajado activamente en el centro urbano impulsando experiencias de organización de los comerciantes indios asentados en el valle 
de Jovel. Posteriormente, tras el movimiento armado chiapaneco de 1994, impulsó el tema de la identidad indígena.

A pesar de ser mayor el número de representantes indígenas de diversos «sectores» y partidos en esta administración, ello tampoco significó una presencia significativa en la función pública, ni mucho menos su mayor implicación en la toma de decisiones del ayuntamiento. Sin embargo, la participación como presidentes u otros cargos en comisiones municipales siguió atestiguando la presencia india.

Ciertamente, la incorporación de indígenas a las comisiones municipales tampoco contribuyó a la construcción de una agenda india dentro de la administración coleta; por el contrario, según refirieron algunos regidores indios, la plana mestiza ocupaba las mejores comisiones, dejando a los regidores indios las que no querían ${ }^{4}$. Aunque dicha dinámica no era nueva, da cuenta de que el proceso de construcción de ciudadanía indígena y los escenarios de inclusión en los espacios de representación, por lo menos en el caso sancristobalense actualmente, se encuentran aterrizados en el plano ideológico-discursivo y atravesados por procesos de «negociación» que poco han contribuido a la construcción de una agenda india.

Al igual que las administraciones de finales de los años noventa, la gestión de Lobato García continuó incorporando a funcionarios indios como presidentes de comisiones municipales, aunque ello no significó que asumieran mayores potestades, por lo que poco pudieron contribuir a la elaboración de una agenda indígena en el municipio. En esta administración se ubicó al indígena Mateo Hernández como presidente de la comisión de panteones, parques y jardines, al Prof. Antonio García como presidente de la comisión de mercados, centros de abasto, rastro y ambulantaje, y al regidor de la facción de convergencia Agustín Gómez como presidente de la comisión de la juventud y el deporte.

Esta presencia se complementó con la integración del regidor Mateo Hernández en la 
comisión de contratación de obras, adquisiciones, arrendamiento y recursos materiales, la comisión de la juventud y el deporte, y la comisión de educación, cultura y recreación. A su vez, el regidor Filiberto Fermín Gómez se integró a la comisión de agua potable y alcantarillado, la comisión de protección al «medio ambiente», y la comisión de alumbrado público y electrificación. Por su parte, el regidor Antonio García únicamente formó parte de la comisión de panteones, parques y jardines, y finalmente Agustín Gómez participó en la comisión de hacienda.

Si bien la presencia de los funcionarios indígenas en la administración municipal se diversificó al incorporarse un número mayor de cargos de procedencia indígena, continuó siendo limitada su intervención en la gestión y, según los propios regidores indios, fundamentalmente los de las facciones de oposición —PVEM y PC—-, sobre todo en los espacios de toma de decisiones.

Aquí mi obligación es cubrir las reuniones, reuniones de cabildo, observar esta administración, pero sabemos que no podemos alcanzar a ver todo, parece que no somos nada en este mundo, en lo que sí estoy luchando es en la parte de la gestoría en diferentes instancias. He platicado con diferentes agentes, representantes que si ellos quieren algo yo puedo ayudar a gestionar y a caminar junto con ellos, es lo que puedo hacer aquí, gran cosa aquí como regidor no puedo hacer nada, y como vuelvo a decir, estoy triste porque no puedo responder a las necesidades de la sociedad, pero en lo que puedo, pues aquí estoy para eso. ${ }^{5}$

A diferencia del discurso indio desde la oposición, los representantes priístas se han ubicado en un terreno más institucional. A pesar de que su presencia tampoco ha implicado mayores potestades en el proceso de toma de decisiones, el discurso de los funcionarios, de alguna u otra manera deja entrever una dinámica construida dentro de la administración coleta.

Bueno, nosotros nos sentimos, pues contentos, porque tenemos la oportunidad de hacer 
algo, aunque no está todo en nuestras manos, porque como es un grupo colegiado, entonces todas las decisiones se toman en conjunto ${ }^{6}$.

Como muestra este testimonio, ha sido común que los funcionarios indios priístas, apelen a la buena voluntad, a su responsabilidad como funcionarios y al trabajo de gestión dentro del ayuntamiento pese a los espacios reducidos que han ocupado en las administraciones sancristobalenses y que han significado prácticamente el mero nombramiento de éstos y no una mayor participación en el ejercicio administrativo y en la toma de decisiones.

Asimismo, hay que señalar que las experiencias de incorporación de los dirigentes indígenas a la escena administrativa municipal continúan caminando en paralelo con coyunturas políticas locales —los procesos migratorios, la organización vecinal indígena, la propia emergencia de 1994 y los procesos electorales locales- muchas veces incompatibles con los procesos de ciudadanización indígena y del propio contexto de visibilización étnico-indígena atestiguado en la otrora ciudad mestiza.

Por ejemplo, durante la administración 2008-2010, la incorporación del dirigente transportista Andrés Gómez López a la planilla del PRD obedeció más a su ubicación en el ramo del transporte regional y en los procesos de «negociación» con la dirigencia perredista municipal, que a un verdadero proceso de inclusión al ejercicio de toma de decisiones, toda vez que ni siquiera formó parte de la planilla perredista municipal. A decir del propio Andrés, su incorporación al ayuntamiento obedeció básicamente a la benevolencia de la dirigencia del PRD, que veía imprescindible la presencia indígena en la dinámica administrativa. No obstante, su presencia también dio cuenta de la delgada frontera existente entre las buenas prácticas ciudadanas y los procesos de cooptación del voto indio:

Pues yo estoy aquí en el ayuntamiento de San Cristóbal, pero la verdad ya había comentado a la dirigencia del PRD que ya no quería seguir participando como PRD, pero pues ellos me dijeron que en estas últimas elecciones [refiriéndose al proceso electoral de 2007] era importante que 
participara. Ellos me dijeron, «Andrés, tú eres indígena, necesitamos que estés participando con nosotros», y pues me convencí y ahora estamos aquí como regidores del PRD ayudando en lo que se puede en el trabajo de gestión de los compañeros indígenas que vienen a plantearnos su situación, su realidad y pues en lo poco que podemos ayudar ahí estamos.

En cuanto a las experiencias de los funcionarios indígenas dentro de la dinámica administrativa, si bien su presencia fue avalada con la incorporación a las diversas comisiones municipales — algunas clave dentro de la dinámica coleta — su participación se redujo a la mera designación institucional, con pocos efectos evidentes, según se observa en el libro de actas de cabildo del ayuntamiento coleto.

Las siguientes palabras del Ing. Filiberto Fermín apuntan hacia una participación dentro de la dinámica administrativa, aunque el planteamiento solamente da cuenta de su contribución en la mecánica del propio ejercicio de gestión:

Bueno, sí, pertenezco a la comisión de agua potable y alcantarillado. Bueno, nosotros participamos como vocal en la junta de gobierno, en donde se aprueban todos los acuerdos que presenta el director. También nosotros podemos presentar propuestas, o sea, para mejorar el servicio, conseguir recursos, implementar algunos recursos a las tarifas. Digamos eso es parte de nuestro trabajo y también estar al tanto de algunas obras que tienen que ver con el agua y con el drenaje, entonces estar al tanto de a ver si se realizan bien y hacer las recomendaciones pertinentes al organismo en caso de que se presenten algunas irregularidades.

Efectivamente, aunque la incorporación de indígenas a cargos municipales —como presidentes u otros - ha sido pregonada por la misma representación india como un «logro» histórico y una respuesta justa a sus reivindicaciones, hay que señalar que, durante la revisión de los libros de acuerdos del cabildo coleto, ha sido común encontrar poca participación, por no decir nula, de los funcionarios indios en el debate administrativo. 


\section{ALGUNAS REFLEXIONES FINALES}

En los últimos catorce años, el proceso de incorporación de la población indígena a la lógica administrativa coleta ha caminado en paralelo a un complejo entramado sociohistórico con efectos evidentes en lo local. Dicho escenario ha involucrado un conjunto de procesos a primera vista diversos: las condiciones de vida, las transformaciones en el ámbito jurídico nacional y estatal, las demandas étnico-indígenas, sus estrategias y formas de negociación étnica, además de los discursos y los símbolos desplegados.

Si bien los líderes indígenas se han incorporado a la arena electoral y al propio ejercicio administrativo coleto como funcionarios públicos - regidores - durante los últimos catorce años en el municipio de San Cristóbal de Las Casas, dicha trayectoria puede derivarse del movimiento étnico de 1994 y de la propia dinámica de organización india construida en la llamada zona norte en pos del derecho a la ciudad.

En ese sentido, debe enfatizarse que la etnicidad se posicionaría como un recurso en constante dinamismo, no como algo fijo o una esencia, sino como un proceso en perpetua construcción. En el caso de los funcionarios indígenas sancristobalenses hay que señalar que, lejos de formar parte de los llamados procesos de reorganización étnica, su participación se ha reducido a la negociación y «transacción» del voto, muchas veces colaborando en la mecánica administrativa pero con pocos resultados evidentes en la agenda gubernamental sobre materia india.

De este modo, las intenciones de re-significar la trayectoria étnico-indígena desde una perspectiva que contribuya a la inclusión en la lógica administrativa municipal quedan anuladas dado que los espacios se construyen en función de contextos discursivos, muchos de ellos desarrollados en las etapas electorales, que poco han contribuido a los procesos de visibilización e incorporación a la mecánica administrativa e incluso al propio contexto 
político-cultural.

Igualmente, la experiencia sancristobalense da cuenta de avances en lo ideológicodiscursivo, en el imaginario del empoderamiento indígena, en las propias experiencias reivindicativas en pro de una ciudadanía diferenciada y en las instituciones democráticas municipales. No obstante, este contexto continúa viéndose influenciado por las prácticas políticas concretas de los participantes, muchas veces inspiradas en la cooptación, el clientelismo y el corporativismo, dinámicas que no están contribuyendo a modificar las instituciones locales para implementar políticas de equidad y propulsar la participación política de los indígenas en el municipio.

Si bien en los últimos catorce años se han cimentado en San Cristóbal de Las Casas otras dinámicas de participación política indígena —miembros del cabildo, integrantes de comisiones municipales, miembros de partidos políticos- estos nuevos roles, en sí mismos, no ofrecen una lectura sobre los alcances y limitaciones de los representantes indígenas una vez situados en los gobiernos locales. De ahí que resulte importante rastrear su presencia en términos de gestión y de participación en la administración local.

Es decir, estos nuevos roles no implican forzosamente que estemos hablando de un «empoderamiento» de la población indígena en la administración local, sino más bien de una etapa de representación producto de caminos contradictorios dirigidos a la búsqueda de una ciudadanía que en muchas ocasiones se ha visto influenciada por formas corporativas de participación dentro de la política local. Estos caminos desiguales de participación, además de las estrategias complejas y novedosas construidas en función de las realidades vividas en la cabecera municipal, nos indican que el debate sobre la gestión de la agenda política y el rol efectivo de los funcionarios dentro de la lógica política administrativa local tienen que seguir en la mesa de discusión. 
BIBLIOGRAFÍA

Assies, Willem y Hans Gundermann, 2007, «Introducción», en Movimientos indígenas y gobiernos locales en América Latina, editado por Assies, Willem y Hans Gundermann, Universidad Católica del Norte, Instituto de Investigaciones Arqueológicas y Museo IIAM, El Colegio de Michoacán, pp. 11-25.

Barth, Fredrik, 1976, Los grupos étnicos y sus fronteras, Fondo de Cultura Económica, México.

Bello, Álvaro, 2004, Etnicidad y ciudadanía en América Latina. La acción colectiva de los pueblos indígenas, CEPAL, Santiago de Chile.

Bengoa, José, 2009, «¿Una segunda etapa de la emergencia indígena en América Latina?», en Cuadernos de Antropología Social, n. 29, FFyL - UBA, Buenos Aires, pp. 7-22.

Clair Ramos, Rosie, 1995, «La democracia y el movimiento indígena en Brasil: reformas posibles en la Constitución de 1988», en Sociedad y Derecho. Indígenas en América Latina, coordinado por Thomas Calvo y Bernardo Méndez, Centro de Estudios Mexicanos y Centroamericanos (CEMCA), México, pp. 155-189.

Freidenberg, Flavia, 2004, «Fracturas sociales y sistema de partidos en Ecuador», en Etnicidad, autonomía y gobernabilidad en América Latina, editado por Martí Puig, Salvador y Josep Ma. Sanahuja, Universidad de Salamanca, Salamanca, pp.101-138.

Gall, Olivia, 2003, «Guerra interétnica y racismo en la historia de Chiapas. Ladinos e indios, miedos y odios», en Chiapas: sociedad, economía, interculturalidad y política, coordinado por Gall, Olivia, Universidad Nacional Autónoma de México, Centro de Investigaciones Interdisciplinarias en Ciencias y Humanidades, México, pp. 57-94. 
Giménez, Gilberto, 2006, «El debate contemporáneo en torno al concepto de etnicidad», en Revista electrónica de Ciencias Sociales, año 1, n. 1, septiembre, Instituto de Investigaciones Sociales, UNAM, México, pp. 129-143.

Gros, Christian, 1998, «Identidades indias, identidades nuevas. Algunas reflexiones a partir del caso colombiano», en Revista Mexicana de Sociología, v. 60, n. 4, octubre-diciembre, Instituto de Investigaciones Sociales, UNAM, México, pp. 181-207.

Gutiérrez Martínez, Daniel, 2008, «Revisitando el concepto de etnicidad: a manera de introducción», en Revisitar la etnicidad. Miradas cruzadas en torno a la diversidad, coordinado por Gutiérrez Martínez, Daniel y Helene Balslev Clausen, Siglo XXI, El Colegio Mexiquense, El Colegio de Sonora, México, pp. 13-40.

Hvostoff, Sophie, 2004, «Indios y coletos: por una relectura de las relaciones interétnicas en San Cristóbal de Las Casas, Chiapas», en Tejiendo historias. Tierra, género y poder en Chiapas, coordinado por Pérez Ruiz, Maya Lorena, Instituto Nacional de Antropología e Historia, Serie Antropológica, México.

Le Bot, Yvon, 2008, «La guerra en el seno de las culturas», en Revisitar la etnicidad: miradas cruzadas en torno a la diversidad, coordinado por Gutiérrez Martínez, Daniel y Helene Balslev Clausen, El Colegio Mexiquense, El Colegio de Sonora, Siglo XXI, México, pp. 83-108.

Martínez Neira, Christian, 2008, «¿Qué son los movimientos étnicos? Las categorías de igualación y diferenciación», en Revisitar la etnicidad: miradas cruzadas en torno a la diversidad, coordinado por Gutiérrez Martínez, Daniel y Helene Balslev Clausen, El Colegio Mexiquense, El Colegio de Sonora, Siglo XXI, México, pp. 71-82. 
Obregón Rodríguez, María Concepción, 2003, Tzotziles, Comisión para el Desarrollo de los Pueblos Indígenas, Programa de Naciones Unidas para el Desarrollo, México.

Restrepo, Eduardo, 2004, Teorías contemporáneas de la etnicidad Stuart Hall y Michel Foucault, Editorial Universidad del Cauca, Popayán.

Río Ruiz, Manuel Ángel, 2002, «Visiones de la etnicidad», en Reis: Revista española de investigaciones sociológicas, n. 98/02, Centro de investigaciones Sociológicas, Madrid, pp. 79-106.

Smith, Anthony, 1997, La identidad nacional, Trama Editorial, Madrid.

Stavenhagen, Rodolfo, 2003, Informe del Relator Especial sobre la situación de los derechos humanos y las libertades fundamentales de los indígenas, Comisión de Derechos Humanos, $59^{\circ}$ período de sesiones, E/CN.4/2003/90, 21 de enero.

Terrén Lalana, Eduardo, 2002, «La etnicidad y sus formas: aproximación a un modelo complejo de la pertenencia étnica», en Papers: Revista de Sociología, n. 66, Departament de Sociologia de la Universitat Autònoma de Barcelona, Barcelona, pp. 45-57.

Wade, Peter, 2000, Raza y etnicidad en Latinoamérica, Abya-Yala, Quito.

\section{NOTAS}

\footnotetext{
${ }^{1}$ Coleto/a: adjetivo coloquial para designar lo perteneciente o relativo a la ciudad de San Cristóbal de Las Casas (Chiapas), o a las personas de ahí naturales (N. del E.).

${ }^{2}$ Entrevista al Prof. Antonio García González, ex regidor de la administración 2005-2007, San Cristóbal de Las Casas, 23 de noviembre de 2009.

${ }^{3}$ Entrevista al Ing. Filiberto Fermín Gómez Rodríguez, ex regidor de la administración 2005-2007, San Cristóbal de Las Casas, 10 de febrero de 2010.

${ }^{4}$ Entrevista al regidor Andrés Gómez López, San Cristóbal de Las Casas, 28 de enero de 2010.
} 
${ }^{5}$ Entrevista a Agustín Gómez Patishtán, ex regidor de la administración 2005-2007, San Cristóbal de Las Casas, 26 de febrero de 2010.

${ }^{6}$ Entrevista al regidor Pascual López Gómez, San Cristóbal de Las Casas, 23 de abril de 2010.

Fecha de recepción: 12 de mayo de 2011.

Fecha de aceptación: 31 de octubre de 2011. 